\title{
Association of Genetic Variants with Isolated Fasting Hyperglycaemia and Isolated Postprandial Hyperglycaemia in a Han Chinese Population
}

\author{
Xiaomu Kong ${ }^{19}$, Jing Hong ${ }^{19}$, Ying Chen ${ }^{2}$, Li Chen ${ }^{3}$, Zhigang Zhao ${ }^{4}$, Qiang Li $^{5}$, Jiapu Ge ${ }^{6}$, Gang Chen ${ }^{7}$, \\ Xiaohui Guo ${ }^{8}$, Juming $\mathrm{Lu}^{9}$, Jianping Weng ${ }^{10}$, Weiping Jia ${ }^{11}$, Linong $\mathrm{Ji}^{12}$, Jianzhong Xiao', \\ Zhongyan Shan ${ }^{13}$, Jie Liu ${ }^{14}$, Haoming Tian ${ }^{15}$, Qiuhe $\mathrm{Ji}^{16}$, Dalong Zhu ${ }^{17}$, Zhiguang Zhou ${ }^{18}$, \\ Guangliang Shan ${ }^{19}$, Wenying Yang ${ }^{1 *}$
}

1 Department of Endocrinology, Key Laboratory of Diabetes Prevention and Control, China-Japan Friendship Hospital, Beijing, China, 2 Department of Bioinformatics, Beijing Genetics Institute, Shenzhen, Guangdong, China, 3 Department of Endocrinology, Qilu Hospital of Shandong University, Jinan, Shandong, China, 4 Department of Endocrinology, Henan Provincial People's Hospital, Zhengzhou, Henan, China, 5 Department of Endocrinology, Second Affiliated Hospital of Harbin Medical University, Harbin, Heilongjiang, China, 6 Department of Endocrinology, Xinjiang Uygur Autonomous Region's Hospital, Urmqi, Xinjiang, China, 7 Department of Endocrinology, Fujian Provincial Hospital, Fuzhou, Fujian, China, 8 Department of Endocrinology, Peking University First Hospital, Beijing, China, 9 Department of Endocrinology, Chinese People's Liberation Army General Hospital, Beijing, China, 10 Department of Endocrinology, Third Affiliated Hospital of Sun Yat-sen University, Guangzhou, Guangdong, China, 11 Department of Endocrinology, Shanghai Jiaotong University Affiliated Sixth People's Hospital, Shanghai, China, 12 Department of Endocrinology, Peking University People's Hospital, Beijing, China, 13 Department of Endocrinology, First Hospital of China Medical University, Shenyang, Liaoning, China, 14 Department of Endocrinology, Shanxi Provincial People's Hospital, Taiyuan, Shanxi, China, 15 Department of Endocrinology, West China Hospital of Sichuan University, Chengdu, Sichuan, China, 16 Department of Endocrinology, Xijing Hospital of Fourth Military Medical University, Xi'an, Shaanxi, China, 17 Department of Endocrinology, Affiliated Drum Tower Hospital of Nanjing University Medical School, Nanjing, Jiangsu, China, 18 Department of Endocrinology, Xiangya Second Hospital, Changsha, Hunan, China, 19 Department of Epidemiology, Peking Union Medical College, Beijing, China

\begin{abstract}
Background: Though multiple single nucleotide polymorphisms (SNPs) associated with type 2 diabetes have been identified, the genetic bases of isolated fasting hyperglycaemia (IFH) and isolated postprandial hyperglycaemia (IPH) were still unclear. In present study, we aimed to investigate the association of genome-wide association study-validated genetic variants and IFH or IPH in Han Chinese.

Methods/Principal Findings: We genotyped 27 validated SNPs in 6,663 unrelated individuals comprising $341 \mathrm{IFH}, 865 \mathrm{IPH}$ 1,203 combined fasting hyperglycaemia and postprandial hyperglycaemia, and 4,254 normal glycaemic subjects of Han ancestry. The distributions of genotype frequencies of FTO, CDKAL 1 and GCKR were significant different between individuals with IFH and those with IPH (SNP( $\left.p_{\text {trend }}\right)$ : rs8050136(0.0024), rs9939609(0.0049), rs7756992(0.0122), rs780094(0.0037)). Risk allele of FTO specifically increased the risk of IFH (rs8050136: OR 1.403 [95\% Cl 1.125-1.750], $p=0.0027 ;$ rs9939609: 1.398 [1.120-1.744], $p=0.0030)$. G allele of CDKAL1 specifically increased the risk of IPH (1.217 [1.092-1.355], $p=0.0004)$. G allele of GCKR increased the risk of IFH (1.167 [0.999-1.362], $p=0.0513)$, but decreased the risk of IPH (0.891 [0.801-0.991], $p=0.0331$ ). In addition, TCF7L2 and KCNQ1 increased the risk of both IFH and IPH. When combined, each additional risk allele associated with IFH increased the risk for IFH by 1.246 -fold $(p<0.0001)$, while each additional risk allele associated with IPH increased the risk for IPH by 1.190 -fold $(p<0.0001)$.

Conclusion/Significance: Our results indicate that genotype distributions of variants from FTO, GCKR, CDKAL1 were different between IPH and IFH in Han Chinese. Variants of genes modulating insulin sensitivity (FTO, GCKR) contributed to the risk of IFH, while variants of genes related to beta cell function (CDKAL1) increase the risk of IPH.
\end{abstract}

Citation: Kong X, Hong J, Chen Y, Chen L, Zhao Z, et al. (2013) Association of Genetic Variants with Isolated Fasting Hyperglycaemia and Isolated Postprandial Hyperglycaemia in a Han Chinese Population. PLoS ONE 8(8): e71399. doi:10.1371/journal.pone.0071399

Editor: Shengxu Li, Tulane School of Public Health and Tropical Medicine, United States of America

Received April 28, 2013; Accepted June 28, 2013; Published August 19, 2013

Copyright: (c) 2013 Kong et al. This is an open-access article distributed under the terms of the Creative Commons Attribution License, which permits unrestricted use, distribution, and reproduction in any medium, provided the original author and source are credited.

Funding: This research project was supported by grants from Chinese Medical Association Foundation and Chinese Diabetes Society (http://www.cma.org.cn/ ensite/), National 973 Program (2011CB504001)(http://www.973.gov.cn/), China Postdoctoral Science Foundation Grant (2012M520200)(http://res. chinapostdoctor.org.cn/Program/Main.html). The funders had no role in study design, data collection and analysis, decision to publish, or preparation of the manuscript.

Competing Interests: The authors have declared that no competing interests exist.

*E-mail: ywying_1010@163.com

9 These authors contributed equally to this work. 


\section{Introduction}

The number of people with diabetes grows worldwide. In the past few decades, China has experienced a dramatic increase in diabetes incidence. According to the recent Chinese National Diabetes and Metabolic Disorders Study (CNDMDS) performed in 2007-2008, about 92.4 million adults $(9.7 \%$ of the adult population) in China have diabetes [1]. Type 2 diabetes is the most common type of diabetes, caused by an interaction of multiple genetic and environmental factors.

Newly diagnosed diabetes generally includes IFH, IPH or combined fasting and postprandial hyperglycaemia $(\mathrm{FH} / \mathrm{PH})$ [2]. According to the DECODE study [3], IFH and IPH accounted for $40.4 \%$ and $28.4 \%$ of newly diagnosed diabetes in Caucasians, respectively. In a study by Yang et al. [1], the prevalence of IPH was reported to be $47 \%$, and that of IFH was $17 \%$ in newly diagnosed cases of diabetes, indicating IPH was the major type of diabetes in Han Chinese, which is a markedly different profile compared to Caucasians.

It was reported that IFH and IPH are derived from isolated impaired fasting glucose and isolated impaired glucose tolerance respectively [4]. Though the etiology of IFH and IPH is unclear, their heterogeneity in clinical manifestation has been well described. A higher level of insulin resistance within the liver is associated with IFH; whereas, impairment of early phase insulin secretion and total insulin secretion in response to glucose were worse in subjects with IPH [2]. Also, there were significant differences in duration, drug therapy, chronic complications and mortality between IFH and IPH subjects [5]. According to the DECODE study [3], postprandial glucose concentrations of subjects with type 2 diabetes were found to be positively associated with incidence of cardiovascular disease, independent of fasting glucose. However, fasting hyperglycaemia was not found to be predictive of the incidence of cardiovascular disease in patients with type 2 diabetes. Therefore, we speculate that IFH and IPH have different genetic etiology.

It has been reported that all subjects with newly diagnosed IFH or IPH ultimately develop fasting hyperglycaemia combined with postprandial hyperglycaemia over the course of decades [4]. In addition, type 2 diabetes patients recruited in hospital-based studies cannot be distinguished to have IFH or IPH when they are first diagnosed. Over the past decade, many studies have attempted to elucidate susceptibility genes for type 2 diabetes. To date, more than 50 genes have been found to be involved in the pathogenesis of type 2 diabetes, mostly in Caucasian populations. However, no susceptible genetic loci of IFH or IPH have been investigated due to difficulties in sampling. It is worthwhile to determine whether common genetic variations play a role in their pathogenesis, as well as to distinguish the common and different genetic basis of IFH and IPH.

In this study, we examined the association of 27 SNPs in GWAS-validated type 2 diabetes susceptible variants [6-18] in patients with IPH or IFH. The study population was a nationally representative cohort of newly diagnosed patients of Han ancestry recruited during CNDMDS conducted in 2007-2008. Further, we compared the difference of genetic basis of IFH and IPH.

\section{Methods}

\section{Ethics Statement}

The study protocol was approved by the Ethics Committee of China-Japan Friendship Hospital in Beijing and was in accordance with Helsinki Declaration II. Written informed consent was obtained from all participants before data collection.

\section{Participants}

All samples were recruited from CNDMDS [1]. After the exclusion of subjects with type 1 diabetes and special type diabetes, a total of 6,663 unrelated Han people from 13 provinces and municipalities participated in the final analysis, including 341 with IFH, 865 with IPH, 1,203 with combined FH/PH, and 4,254 control subjects. Each participant received a standard $75 \mathrm{~g}$ oral glucose tolerance test (OGTT). Type 2 diabetes was defined by 1999 WHO criteria. IFH was defined as fasting plasma glucose $($ FPG $) \geq 7 \mathrm{mmol} / \mathrm{l}(126 \mathrm{mg} / \mathrm{dl})$ and 2-hour plasma glucose in OGTT $(2 \mathrm{~h} \mathrm{PG})<11.1 \mathrm{mmol} / \mathrm{l}(200 \mathrm{mg} / \mathrm{dl})$. IPH was defined as FPG $<7.0 \mathrm{mmol} / \mathrm{l}$ and $2 \mathrm{~h} \mathrm{PG} \geq 11.1 \mathrm{mmol} / \mathrm{l}$. Combined FH/ $\mathrm{PH}$ was defined as $\mathrm{FPG} \geq 7 \mathrm{mmol} / \mathrm{l}$ and $2 \mathrm{~h} \mathrm{PG} \geq 11.1 \mathrm{mmol} / \mathrm{l}$. The inclusion criteria for normoglycaemic control were: 1) age over 40 years; 2) normal glucose regulation in OGTT according to WHO criteria (FPG $<6.1 \mathrm{mmol} / \mathrm{l}(110 \mathrm{mg} / \mathrm{dl})$ and $2 \mathrm{~h} \mathrm{PG}$ $<7.8 \mathrm{mmol} / \mathrm{l}(140 \mathrm{mg} / \mathrm{dl})$ ); 3) no diabetes history or family history of diabetes; 4) $\mathrm{BMI}<28 \mathrm{~kg} / \mathrm{m}^{2}$; 5) normal blood pressure (systolic blood pressure $<140 \mathrm{mmHg}$ and diastolic blood pressure $<90 \mathrm{mmHg}$ ); 6) normal blood lipid levels (triglycerides (TG) $<1.7 \mathrm{mmol} / \mathrm{l}$ and high density lipoprotein-cholesterol $\geq 1.0 \mathrm{mmol} / \mathrm{l}$ ). Clinical characteristics of the study groups are shown in Table 1 . Normally distributed data are shown as mean \pm SD while non-Gaussian data are shown as median and interquartile range. Non-fatal cardiovascular diseases were determined as previously reported [1].

\section{Clinical examinations}

Anthropometric and biochemical characteristics were carefully examined. Height, weight, waist circumference (WC) and hip circumference were measured with subjects lightly clothed, from which BMI and waist-hip-ratio were calculated. Without glucoselowering treatment, subjects were requested to fast from food for more than 10 hours and given a standard $75 \mathrm{~g}$ OGTT the next day. Blood samples were drawn at 0,30 and 120 minutes after OGTT to measure plasma glucose and serum insulin concentrations. Serum insulin was measured by double-antibody radioimmunoassay. HOMA was used to estimate insulin resistance (HOMA-IR: fasting serum insulin $(\mathrm{mU} / \mathrm{l}) \times \mathrm{FPG}(\mathrm{mmol} / \mathrm{l}) /$ 22.5) and beta cell function (HOMA-B: fasting serum insulin $(\mathrm{mU} / \mathrm{l}) \times 20 /(\mathrm{FPG}(\mathrm{mmol} / \mathrm{l})-3.5))$. Insulinogenic index $\left(\Delta \mathrm{I}_{30} /\right.$ $\Delta \mathrm{G}_{30}$ ) was calculated from fasting and 30 -min serum insulin (mU/ l) and plasma glucose $(\mathrm{mmol} / \mathrm{l})$ during OGTT using $\left(\mathrm{Ins}_{30}-\mathrm{Ins}_{0}\right) /$ $\left(\mathrm{Glu}_{30}-\mathrm{Glu}_{0}\right)$. The compensation of beta cells to insulin resistance was calculated using $\Delta \mathrm{I}_{30} / \Delta \mathrm{G}_{30} /$ HOMA-IR. Matsuda index $(\mathrm{ISIm})$ was calculated as $10,000 /\left(\mathrm{Glu}_{0}(\mathrm{mg} / \mathrm{dl}) \times \operatorname{Ins}_{0}(\mathrm{mU} / \mathrm{l}) \times\right.$ mean glucose OGTT $(\mathrm{mg} / \mathrm{dl}) \times$ mean insulin $(\mathrm{mU} / \mathrm{l}))^{1 / 2}$.

Total cholesterol, TG, high density lipoprotein-cholesterol and low density lipoprotein-cholesterol in fasting serum were tested using an automatic biochemical analyzer (Olympus, Tokyo, Japan).

Joint study of IPH associated SNPs, including TCF7L2, CDKAL1, KCNQ1, PRC1, TP53INP1 and GCKR, shows that individuals with IPH carry more risk alleles from the above loci than controls $\left(p_{\text {trend }}<0.0001\right.$; Figure 2A). Risk of IPH increased $19.0 \%$ for each additional risk allele carried (1.190 [1.120-1.263] per allele, $p<0.0001$; Figure 2B). In SNPs associated with IPH, the numbers of risk alleles carried in IFH subjects were not significantly more than that in the control group $\left(p_{\text {trend }}=0.2752\right.$; Figure 2A); and the number of risk alleles did not increase the risk of IFH (1.053 [0.963-1.150] per allele, $p=0.2564$; Figure 2C). 
Table 1. Clinical characteristics of study population.

\begin{tabular}{|c|c|c|c|c|}
\hline & & Isolated fasting & Isolated postprandial & All newly diagnosed \\
\hline & control & hyperglycaemia & hyperglycaemia & type 2 diabetes \\
\hline Samples $(n)$ & 4,254 & 341 & 865 & 2,409 \\
\hline Male/Female $(n)$ & $1,382 / 2,872$ & $175 / 166$ & $382 / 483$ & $1,062 / 1,347$ \\
\hline Age (years) & $50.69 \pm 8.38$ & $51.19 \pm 12.58$ & $56.17 \pm 11.52$ & $55.20 \pm 11.60$ \\
\hline BMI $\left(\mathbf{k g} / \mathbf{m}^{2}\right)$ & $23.07(21.28,24.77)$ & $25.26(23.03,28.56)$ & $25.77(23.52,28.13)$ & $25.85(23.62,28.33)$ \\
\hline Fasting plasma glucose ( $\mathrm{mmol} / \mathrm{l})$ & $5.02(4.68,5.40)$ & $7.53(7.20,8.26)$ & $6.00(5.45,6.50)$ & $7.49(6.41,9.16)$ \\
\hline 30min plasma glucose (mmol/l) & $8.10(7.00,9.20)$ & $11.10(9.20,12.98)$ & $11.30(9.64,12.79)$ & $12.27(10.32,14.55)$ \\
\hline $2 \mathrm{~h}$ plasma glucose $(\mathrm{mmol} / \mathrm{l})$ & $5.76(4.90,6.60)$ & $8.41(6.71,9.70)$ & $12.61(11.76,14.18)$ & $13.70(11.72,17.19)$ \\
\hline Fasting serum insulin (mU/l) & $6.31(4.90,8.45)$ & $9.10(6.14,12.30)$ & $8.16(5.93,11.66)$ & $8.80(6.12,12.62)$ \\
\hline 30min serum insulin (mU/I) & $33.09(20.92,52.64)$ & $28.18(17.05,47.99)$ & $25.84(15.11,43.80)$ & $20.67(11.87,36.86)$ \\
\hline $2 \mathrm{~h}$ serum insulin $(\mathrm{mU} / \mathrm{l})$ & $22.37(13.94,34.96)$ & $29.26(17.97,49.56)$ & $50.96(26.56,90.70)$ & $33.44(19.07,61.04)$ \\
\hline HOMA-IR & $1.40(1.06,1.90)$ & $3.22(2.12,4.47)$ & $2.15(1.49,3.10)$ & $3.07(1.98,4.61)$ \\
\hline HОМА-B (\%) & $85.55(61.09,125.08)$ & $42.47(29.30,61.20)$ & $71.58(47.89,102.95)$ & $45.27(27.62,73.88)$ \\
\hline ISIm & $8.38(6.19,11.34)$ & $4.35(3.08,6.32)$ & $4.66(2.97,6.82)$ & $4.13(2.78,6.04)$ \\
\hline Insulinogenic index $\left(\Delta \mathbf{I}_{30} / \Delta \mathbf{G}_{30}\right)$ & $9.40(5.03,17.31)$ & $4.74(2.10,11.68)$ & $3.36(1.78,6.78)$ & $2.68(1.29,5.98)$ \\
\hline $\begin{array}{l}\text { Prevalence of cardiovascular } \\
\text { diseases }\left(\%, n_{\text {total }} n_{\text {affected }}\right)\end{array}$ & $3.25 \%, 3,422 / 115$ & $6.72 \%, 250 / 18$ & $11.72 \%, 625 / 83$ & $11.18 \%, 1,967 / 220$ \\
\hline
\end{tabular}

Data are shown as mean \pm SD for normally distributed values, median (interquartile range) for non-normally distributed values, or $n(\%)$.

doi:10.1371/journal.pone.0071399.t001

Table 2. SNPs significantly associated with isolated fasting hyperglycemia in Hans.

\begin{tabular}{|c|c|c|c|c|c|c|}
\hline \multirow[b]{2}{*}{ Gene } & \multirow[b]{2}{*}{ SNP } & \multicolumn{2}{|c|}{ Minor/major } & \multirow{2}{*}{$\frac{\text { Allelic }}{\text { association }^{\text {b }}}$} & \multirow{2}{*}{$\frac{\text { Genotypic }}{\text { associationc }^{c}}$} & \multirow{2}{*}{$\frac{\text { Genotypic }}{\text { association }^{d}}$} \\
\hline & & allele $^{a}$ & & & & \\
\hline \multirow[t]{3}{*}{ TCF7L2 } & rs7903146 & $\mathrm{T} / \mathrm{C}$ & OR $(95 \% \mathrm{Cl})$ & $1.524(1.074,2.164)$ & $1.454(1.019,2.075)$ & $1.503(1.023,2.208)$ \\
\hline & & & $p$ & 0.0176 & 0.0390 & 0.0379 \\
\hline & & & Empirical $p$ & 0.4236 & & \\
\hline \multirow[t]{3}{*}{ KCNQ1 } & rs2237895 & $\mathrm{C} / \mathrm{A}$ & OR (95\%Cl) & $1.201(1.008,1.431)$ & $1.211(1.016,1.444)$ & $1.194(0.991,1.439)$ \\
\hline & & & $p$ & 0.0401 & 0.0327 & 0.0617 \\
\hline & & & Empirical $p$ & 0.7003 & & \\
\hline \multirow[t]{3}{*}{ CDKN2BAS } & rs10811661 & $\mathrm{C} / \mathbf{T}$ & OR $(95 \% \mathrm{Cl})$ & $1.189(1.016,1.392)$ & $1.183(1.011,1.384)$ & $1.220(1.034,1.441)$ \\
\hline & & & $p$ & 0.0310 & 0.0358 & 0.0188 \\
\hline & & & Empirical $p$ & 0.6034 & & \\
\hline \multirow[t]{3}{*}{ FTO } & rs8050136 & $A / C$ & OR $(95 \% \mathrm{Cl})$ & $1.405(1.129,1.750)$ & $1.403(1.125,1.750)$ & $1.263(0.995,1.604)$ \\
\hline & & & $p$ & 0.0023 & 0.0027 & 0.0554 \\
\hline & & & Empirical $p$ & 0.0629 & & \\
\hline \multirow[t]{3}{*}{ FTO } & rs9939609 & $\mathbf{A} / \mathrm{T}$ & OR $(95 \% \mathrm{Cl})$ & $1.394(1.119,1.735)$ & $1.398(1.120,1.744)$ & $1.253(0.987,1.590)$ \\
\hline & & & $p$ & 0.0029 & 0.0030 & 0.0642 \\
\hline & & & Empirical $p$ & 0.0819 & & \\
\hline \multirow[t]{3}{*}{ GCKR } & rs780094 & $\mathbf{G} / \mathrm{A}$ & OR (95\%Cl) & $1.171(1.002,1.369)$ & $1.167(0.999,1.362)$ & $1.166(0.989,1.374)$ \\
\hline & & & $p$ & 0.0476 & 0.0513 & 0.0678 \\
\hline & & & Empirical $p$ & 0.7562 & & \\
\hline
\end{tabular}

${ }^{\text {a }}$ Risk alleles for type 2 diabetes in the Caucasian descent population are denoted in bold. OR and $95 \% \mathrm{Cl}$ are reported for the allele with higher type 2 diabetes risk previously reported for Caucasians using $\chi^{2}$ or an additive model in logistic regression.

${ }^{\mathrm{b} C}$ Comparison of the allelic distribution between isolated fasting hyperglycemia and controls.

'Comparison of the genotype distribution between isolated fasting hyperglycemia and controls after adjusting for region, age and gender.

${ }^{\mathrm{d} C}$ Comparison of the genotype distribution between isolated fasting hyperglycemia and controls after adjusting for region, age, gender and BMI.

Associations of the rest SNPs with isolated fasting hyperglycemia are shown in Table S4.

Empirical $p$ values were calculated through 1,000 permutations. $p$ values $<0.05$ are shown in bold.

doi:10.1371/journal.pone.0071399.t002 
A
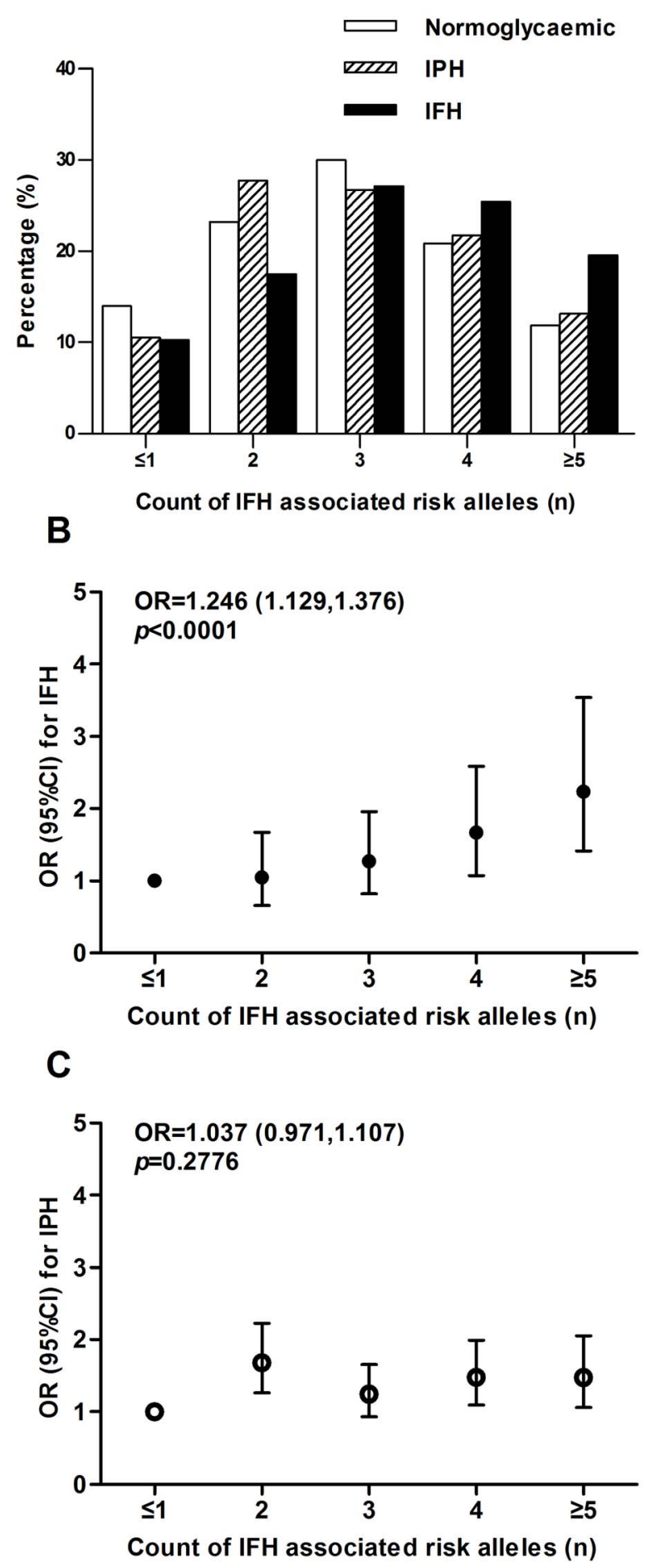

Figure 1. Combined effects of increasing numbers of isolated fasting hyperglycaemia-associated risk alleles for TCF7L2, CDKN2BAS, KCNQ1, FTO and GCKR. A: The isolated fasting hyperglycaemia-associated risk allele distribution in controls, participants with isolated fasting hyperglycaemia and isolated postprandial hyperglycaemia. Each additional risk allele increased the risk of isolated fasting hyperglycaemia by 1.24 -fold $(p<0.0001)$ (B) but not the risk of isolated postprandial hyperglycaemia (C). OR and $95 \% \mathrm{Cl}$ plotted on the $y$-axis for the corresponding number of risk alleles on the $x$-axis (black circles $=$ risk of isolated fasting hyperglycaemia; white circles $=$ risk of isolated postprandial hyperglycaemia). B: Participants harboring five or more risk alleles had a 2.23 -fold increased risk for isolated fasting hyperglycaemia $(p<0.0001)$ compared with the reference group. C: However, risk for isolated postprandial hyperglycaemia was not increased as risk allele number increased. IFH, isolated fasting hyperglycemia; IPH, isolated postprandial hyperglycemia. doi:10.1371/journal.pone.0071399.g001

\section{Genotyping}

Genomic DNA samples were isolated from the peripheral blood using a DNA extraction kit. Genotyping was accomplished with Illumina GoldenGate Indexing assay (Illumina Inc., San Diego, USA), used according to the manufacturer's instructions. We selected 31 common SNPs from 29 type 2 diabetes susceptible loci in the Caucasian population, including KCNQ1 (rs2237895, rs231362), CDKAL1 (rs7756992), CDKN2BAS (rs10811661), TCF7L2 (rs7903146), HHEX (rs1111875), TCF2 (rs7501939), WFS1 (rs10010131), CDC123/CAMK1D (rs12779790), MTNR1B (rs10830963), FTO (rs8050136, rs9939609), ADAMTS9 (rs4607103), JAZF1 (rs864745), TSPAN8/LGR5 (rs7961581), TP53INP1 (rs896854), PRC1 (rs8042680), GCKR (rs780094), ZFAND6 (rs11634397), BCL11A (rs243021), KLF14 (rs972283), ZBED3 (rs4457053), HNF1A (rs7957197), CENTD2 (rs1552224), NOTCH2 (rs10923931), CHCHD9 (rs13292136), PPARG (rs 1801282), THADA (rs7578597), SLC30A8 (rs13266634), HMGA2 (rs 1531343), DUSP9 (rs5945326) [6-18]. Only the SNPs with Genotyping success rate $>90 \%$ were investigated (rs231362, rs13266634, rs1531343 and rs5945326 were excluded from the study for their genotyping success rate was lower than $90 \%$ ). The overall mean calling rate of $27 \mathrm{SNPs}$ was $99.47 \%$. The concordance rate based on 229 genotyping duplication was $100 \%$.

\section{Statistical analyses}

Hardy Weinberg equilibrium test was done for each SNP by $\chi^{2}$ test before further analysis. Difference in allele frequency was determined by $\chi^{2}$ test, with OR and $95 \%$ CI estimated.

The association study was performed assuming an additive model. Genotype distributions between case and control were compared using logistic regression adjusted for geographical region, age and gender. OR and 95\% CI were calculated before and after adjustment for BMI. Quantitative characteristics of normoglycaemic or newly diagnosed type 2 diabetes individuals were analyzed by linear regression adjusted for geographical region, age and gender, while BMI was adjusted when appropriate (apart from anthropometric traits including height, weight, WC, hip circumference, waist-hip-ratio and BMI), and regression coefficients $(\beta \mathrm{s})$ were determined. All non-Gaussian distributed quantitative traits were subjected to natural logarithm transformation to approximately approach normal distribution. The association studies were adjusted for multiple testing by a permutation step of 1,000 times using the PLINK analysis software. $\chi^{2}$ test and Cochran-Armitage test was used to analyze differences of risk allele frequency and genotype distribution between IFH and IPH.

In order to determine the joint effects of associated SNPs $($ IFH associated SNPs included KCNQ1, CDKN2BAS, TCF7L2, FTO (rs8050136), GCKR, except FTO (rs9939609) which was from the same linkage disequilibrium block with rs8050136; IPH associated SNPs included KCNQ1, CDKAL1, TCF7L2, TP53INP1, PRC1, $G C K R$ ) on the risk of IFH and IPH, we categorized the individuals based on risk alleles they carried, as previous reported [19]. The analysis included individuals for whom genotypes of involved SNPs were available (sample size for IFH SNPs was 291/767/ 4060 (IFH/IPH/Control); sample size for IPH SNPs was 293/ $771 / 4053)$. Considering the association results, we defined the $\mathrm{G}$ allele of rs780094 as a risk allele of IFH SNPs, while A allele was 
Table 3. SNPs significantly associated with isolated postprandial hyperglycemia in Hans.

\begin{tabular}{|c|c|c|c|c|c|c|}
\hline \multirow[b]{2}{*}{ Gene } & \multirow[b]{2}{*}{ SNP } & \multicolumn{2}{|c|}{ Minor/major } & \multirow{2}{*}{$\begin{array}{l}\text { Allelic } \\
\text { association }^{\text {b }}\end{array}$} & \multirow{2}{*}{$\frac{\text { Genotypic }}{\text { association }^{c}}$} & \multirow{2}{*}{$\frac{\text { Genotypic }}{\text { association }^{\text {d }}}$} \\
\hline & & allele $^{a}$ & & & & \\
\hline \multirow[t]{3}{*}{ TCF7L2 } & rs7903146 & $T / C$ & OR (95\%Cl) & $1.507(1.187,1.914)$ & $1.485(1.158,1.906)$ & $1.438(1.080,1.888)$ \\
\hline & & & $p$ & 0.0007 & 0.0019 & 0.0123 \\
\hline & & & Empirical $p$ & 0.0230 & & \\
\hline \multirow[t]{3}{*}{ KCNQ1 } & rs2237895 & $\mathrm{C} / \mathrm{A}$ & OR (95\%Cl) & $1.154(1.030,1.294)$ & $1.153(1.025,1.296)$ & $1.181(1.038,1.343)$ \\
\hline & & & $p$ & 0.0137 & 0.0174 & 0.0115 \\
\hline & & & Empirical $p$ & 0.3317 & & \\
\hline \multirow[t]{3}{*}{ GCKR } & rs780094 & $\mathbf{G} / \mathrm{A}$ & OR (95\%Cl) & $0.904(0.815,1.003)$ & $0.891(0.801,0.991)$ & $0.895(0.796,1.006)$ \\
\hline & & & $p$ & 0.0574 & 0.0331 & 0.0627 \\
\hline & & & Empirical $p$ & 0.8182 & & \\
\hline \multirow[t]{3}{*}{ CDKAL1 } & rs7756992 & $A / \mathbf{G}$ & OR $(95 \% \mathrm{Cl})$ & $1.209(1.089,1.342)$ & $1.217(1.092,1.355)$ & $1.233(1.095,1.389)$ \\
\hline & & & $p$ & 0.0004 & 0.0004 & 0.0005 \\
\hline & & & Empirical $p$ & 0.0180 & & \\
\hline \multirow[t]{3}{*}{ TP53INP1 } & rs896854 & $A / G$ & OR $(95 \% \mathrm{Cl})$ & $1.109(0.996,1.235)$ & $1.129(1.009,1.263)$ & $1.099(0.971,1.245)$ \\
\hline & & & $p$ & 0.0601 & 0.0348 & 0.1360 \\
\hline & & & Empirical $p$ & 0.8282 & & \\
\hline \multirow[t]{3}{*}{ PRC1 } & rs 8042680 & C/A & OR $(95 \% \mathrm{Cl})$ & $1.709(1.045,2.793)$ & $1.758(1.063,2.908)$ & $1.555(0.917,2.639)$ \\
\hline & & & $p$ & 0.0307 & 0.0279 & 0.1016 \\
\hline & & & Empirical $p$ & 0.6164 & & \\
\hline \multirow[t]{3}{*}{ HHEX } & rs1111875 & $\mathbf{G} / \mathrm{A}$ & OR $(95 \% \mathrm{Cl})$ & $1.105(0.987,1.238)$ & $1.102(0.981,1.238)$ & $1.151(1.013,1.309)$ \\
\hline & & & $p$ & 0.0838 & 0.1027 & 0.0309 \\
\hline & & & Empirical $p$ & 1.0000 & & \\
\hline
\end{tabular}

a Risk alleles for type 2 diabetes in the Caucasian descent population are denoted in bold. OR and $95 \% \mathrm{Cl}$ are reported for the allele with higher type 2 diabetes risk previously reported for Caucasians using $\chi 2$ or an additive model in logistic regression.

${ }^{b}$ Comparison of the allelic distribution between isolated postprandial hyperglycemia and controls.

'Comparison of the genotype distribution between isolated postprandial hyperglycemia and controls after adjusting for region, age and gender.

${ }^{\mathrm{d} C}$ Comparison of the genotype distribution between isolated postprandial hyperglycemia and controls after adjusting for region, age, gender and BMI.

Associations of the rest SNPs with isolated postprandial hyperglycemia are shown in Table S5.

Empirical $p$ values were calculated through 1,000 permutations. $p$ values $<0.05$ are shown in bold.

doi:10.1371/journal.pone.0071399.t003

the risk allele for IPH SNPs. Individuals were grouped based on the amount of risk alleles they carried. For the joint study of IFH associated SNPs, individuals were divided into 5 groups $(\leq 1,2,3$, $4, \geq 5)$, while there were 6 groups for IPH SNP analysis $(\leq 3,4,5$, $6,7, \geq 8)$. The group carrying fewest risk alleles was considered as reference $(\mathrm{OR}=1)$. Effective sizes for every unit increase in the number of risk alleles on IFH or IPH were calculated after adjustment for geographical region, age and gender using a logistic regression model.

All statistical analyses were performed using either SAS for Windows (version 9.2; SAS Institute, Cary, NC) or PLINK software (v1.05). All $p$ values $<0.05$ (two-tailed) were considered statistically significant.

Power calculations were performed using Quanto software (available at http://hydra.usc.edu/gxe/) and shown in Table S7. Power was calculated using the ORs reported in the original studies [6-18] as well as sample size and minor allele frequencies (MAF) in the present study. An overall diabetes risk of 9.7\% [1] among Chinese was used in power analysis of newly diagnosed type 2 diabetes. In addition, for power calculation of IFH and IPH, overall IFH and IPH risk $(1.65 \%, 4.56 \%)$ were calculated from $9.7 \%$ multiplying by the proportion of IFH and IPH in newly diagnosed type 2 diabetes $(17 \%, 47 \%)[1]$.

\section{Results}

Association study of 27 SNPs and newly diagnosed type 2 diabetes in Han Chinese

All SNPs were within Hardy-Weinberg equilibrium in controls ( $p>0.05$; Table S1). TCF7L2, CDKN2BAS, CDKAL1, KCNQ1, HHEX, TCF2, CENTD2 and TP53INP1 showed associations with type 2 diabetes (ORs ranged between 1.089-1.385, $p$ value ranged between $4.112 \times 10^{-7}-0.0311$; Table S2). Three of the eight SNPs remained significant after adjustment for multiple comparisons $(p$ value ranged between $0.0020-0.0080$; Table S2).

The association study of SNPs and type 2 diabetes related quantitative traits showed that subjects carrying the CDKAL1 risk allele had higher plasma glucose $30 \mathrm{~min}$ in OGTT $(\beta=0.01669$ per $\mathrm{G}$ allele for $\operatorname{lnFPG} ; p=0.0006)$. The correlation remained after multiple test correction (Empirical $p=0.0140$ ). In cases of newly diagnosed type 2 diabetes, subjects carrying the $K C N Q 1$ risk allele had lower BMI $(\beta=-0.01738$ per for $\mathrm{C}$ allele for $\operatorname{lnBMI}$; $p=0.0001)$, and this correlation remained after multiple test correction (Empirical $p=0.0070$ ) (Table S3).

\section{Clinical characteristics of individuals with IFH and IPH}

As shown in Table 1, individuals with IFH had higher FPG, liver insulin resistance (evaluated by HOMA-IR and ISIm) and 
A

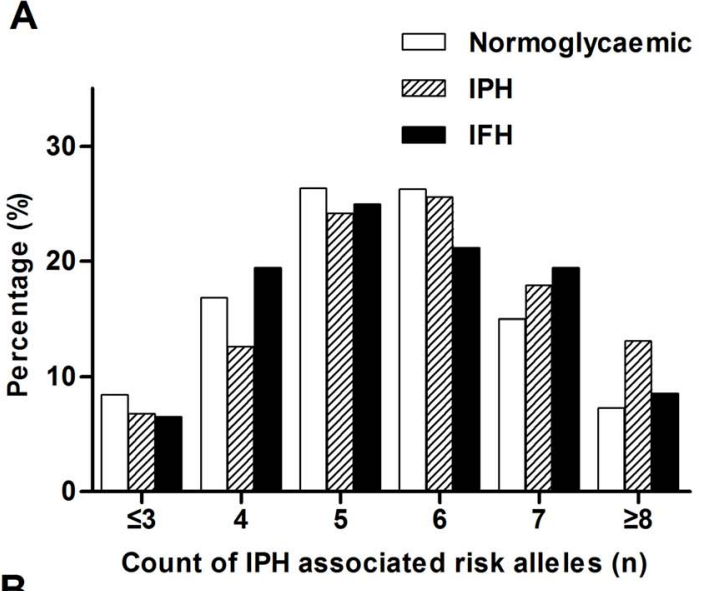

B

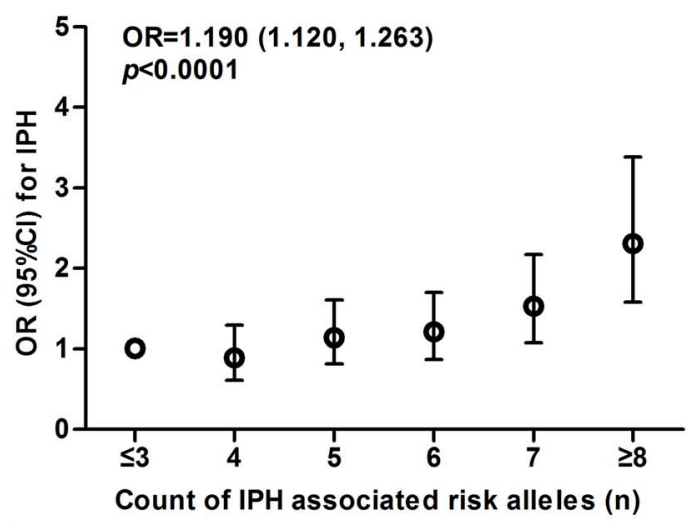

C

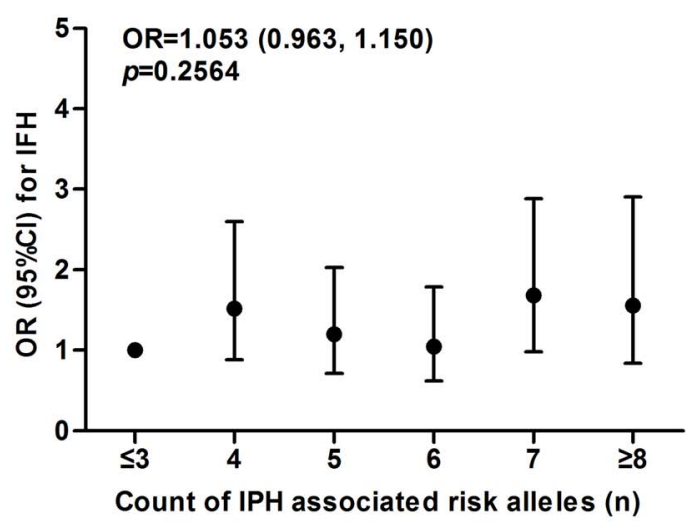

Figure 2. Combined effects of increasing numbers of isolated postprandial hyperglycaemia-associated risk alleles from TCF7L2, CDKAL1, KCNQ1, PRC1, TP53INP1 and GCKR. A: The isolated postprandial hyperglycaemia-associated risk allele distribution in controls, participants with isolated fasting hyperglycaemia and isolated postprandial hyperglycaemia. Each additional risk allele increased the risk of isolated postprandial hyperglycaemia by 1.19 -fold $(p<0.0001)$ (B) but not the risk of isolated fasting hyperglycaemia (C). OR and $95 \% \mathrm{Cl}$ were plotted on the $y$-axis for the corresponding number of risk alleles on the $\mathrm{x}$-axis (black circles = risk of isolated fasting hyperglycaemia; white circles $=$ risk of isolated postprandial hyperglycaemia). $\mathrm{B}$ : Participants harboring eight or more risk alleles had a 2.31-fold increased risk for isolated postprandial hyperglycaemia $(p<0.0001)$ compared with the reference group. C: However, risk for isolated fasting hyperglycaemia was not increased as risk allele number increasing. IFH, isolated fasting hyperglycemia; IPH, isolated postprandial hyperglycemia.

doi:10.1371/journal.pone.0071399.g002 better early phase insulin secretion ability (evaluated by $\Delta \mathrm{I}_{30}$ / $\Delta \mathrm{G}_{30}$ ) compared to subjects with IPH. 2h PG of individuals with IPH was higher, as well as higher prevalence of cardiovascular disease. HOMA-B was lower in IFH than IPH, which was calculated from FPG and fasting insulin level.

\section{Association study of SNPs and IFH in Hans}

Table 2 shows that TCF7L2, CDKN2BAS, KCNQ1, FTO and GCKR are significantly associated with IFH (OR ranged between $1.171-1.524 ; p$ value ranged between $0.0023-0.0476)$. When adjusted for region, age and gender, TCF7L2 and FTO (rs8050136, rs9939609) risk alleles conferred 1.454-, 1.403- and 1.398-fold increased risk of IFH. After further adjustment for BMI, the significant association between IFH with TCF7L2 or $C D K N 2 B A S$ remained (rs7903146: 1.503 [1.023-2.208], $p=0.0379 ;$ rs10811661: 1.220 [1.034-1.441], $p=0.0188$ ), while KCNQ1 was no longer associated with IFH (1.194 [0.991-1.439], $p=0.0617)$. SNPs in FTO were not associated with IFH after adjustment for BMI (rs8050136: 1.263 [0.995-1.604], $p=0.0554$; rs9939609: 1.253 [0.987-1.590], $p=0.0642)$ with a reduction in OR values.

Joint study of IFH associated SNPs, including TCF7L2, $C D K N 2 B A S, K C N Q 1, F T O$ and GCKR, shows that individuals with IFH carry more risk alleles from the above loci than controls $\left(p_{\text {trend }}\right.$ $<0.0001$; Figure 1A). Risk of IFH increased $24.6 \%$ for each additional risk allele carried (1.246 [1.129-1.376] per allele, $p<0.0001$; Figure 1B). However, individuals with IPH did not carry more risk alleles than controls $\left(p_{\text {trend }}=0.2208\right.$; Figure $\left.1 \mathrm{~A}\right)$. The number of risk alleles did not increase the risk of IPH (1.037 [0.971-1.107] per allele, $p=0.2776$; Figure 1C).

\section{Association study of SNPs and IPH in Han Chinese}

Table 3 shows that TCF7L2, CDKAL1, KCNQ1, and PRC1 are significantly associated with IPH (OR ranged between 1.154$1.709, p$ value ranged between $0.00038-0.03482$ ). Another two SNPs, TP53INP1 and GCKR, were also found to be associated with IPH after adjustment for region, age and gender (rs896854: 1.129 [1.009-1.263], $p=0.0348 ; \quad$ rs780094: 0.891 [0.801-0.991], $p=0.0331$. The PRC1 and TCF7L2 risk alleles conferred a 1.758- and 1.485-fold of increased risk of IPH, respectively. Risk alleles in CDKAL1, KCNQ1 and TP53INP1 conferred a 1.217-, 1.153- and 1.129-fold of increased risk of IPH, respectively. Noticeably, carriers of the validated type 2 diabetes risk allele $G$ of GCKR showed lower risk of IPH; therefore the A allele of GCKR was defined as the risk allele in further joint study. TCF7L2, CDKAL1 and KCNQ1 remained associated with IPH after adjustment for BMI (rs7903146: 1.438 [1.080-1.888], $p=0.0123 ; \quad$ rs7756992: $1.233 \quad[1.095-1.389], \quad p=0.0005$; rs2237895: 1.181 [1.038-1.343], $p=0.0115)$. GCKR, TP53INP1 and PRC1 were not associated with IPH after adjustment for BMI (rs780094: 0.895 [0.796-1.006], $p=0.0627$; rs896854: 1.099 [0.971-1.245], $\quad p=0.1360 ; \quad$ rs8042680: 1.555 [0.917-2.639], $p=0.1016)$.

\section{Difference of allelic frequencies and genotype} distributions between IFH and IPH

Among all tested SNPs, risk allele frequencies of SNPs in FTO and GCKR in IFH were much higher than those in IPH (A allele of rs8050136, $p=0.0026$; A allele of rs9939609, $p=0.0053$; $\mathrm{G}$ allele of rs780094, $p=0.0043$; Table 4). However risk allele frequencies of SNPs in CDKAL1 were much lower in IFH subjects than IPH subjects (G allele of rs7756992, $p=0.0103$ ). Genotype distributions of these SNPs between IFH and IPH were significantly different 
Table 4. SNPs showed significant differences in risk allele frequency and genotype distribution between isolated fasting hyperglycemia and isolated postprandial hyperglycemia.

\begin{tabular}{|c|c|c|c|c|c|c|c|c|c|c|}
\hline \multirow[b]{3}{*}{ Gene } & \multirow{2}{*}{\multicolumn{2}{|c|}{ Minor/major }} & \multirow{2}{*}{\multicolumn{2}{|c|}{$\begin{array}{l}\text { Risk allele } \\
\text { frequency }\end{array}$}} & \multirow{2}{*}{\multicolumn{2}{|c|}{$\frac{\chi^{2} \text { test of risk }}{\text { allele frequency }}$}} & \multirow{2}{*}{\multicolumn{2}{|c|}{$\begin{array}{l}\text { Genotype distribution } \\
\text { (BB/Bb/bb) }\end{array}$}} & \multirow{2}{*}{\multicolumn{2}{|c|}{$\begin{array}{l}\text { Cochran-Armitage trend test } \\
\text { of genotype distribution }\end{array}$}} \\
\hline & & & & & & & & & & \\
\hline & SNP & allele $^{a}$ & IFH & IPH & $\chi^{2}$ & $\mathbf{p}$ & IFH & IPH & $\mathbf{z}$ & Two-tails $p$ \\
\hline FTO & rs8050136 & $\mathbf{A} / \mathbf{C}$ & 0.153 & 0.109 & 9.0651 & 0.0026 & $4 / 96 / 240$ & $12 / 163 / 687$ & -3.0300 & 0.0024 \\
\hline FTO & rs9939609 & $\mathbf{A} / \mathrm{T}$ & 0.153 & 0.111 & 7.7725 & 0.0053 & $4 / 96 / 241$ & $12 / 168 / 684$ & -2.8116 & 0.0049 \\
\hline GCKR & rs780094 & $\mathbf{G} / \mathrm{A}$ & 0.515 & 0.450 & 8.1464 & 0.0043 & $89 / 172 / 79$ & $166 / 446 / 252$ & -2.8990 & 0.0037 \\
\hline CDKAL1 & rs7756992 & $\mathrm{A} / \mathbf{G}$ & 0.510 & 0.568 & 6.5872 & 0.0103 & $94 / 160 / 87$ & $286 / 405 / 169$ & 2.5059 & 0.0122 \\
\hline
\end{tabular}

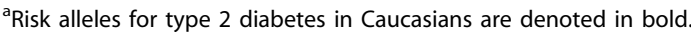

Allelic frequencies between isolated fasting hyperglycemia and isolated postprandial hyperglycemia were compared using $\chi 2$ test.

Genotype distributions are shown as the counts of three genotypes (BB, Bb, bb). B, risk allele; b, non-risk allele. Genotype distributions between isolated fasting hyperglycemia and isolated postprandial hyperglycemia were compared using Cochran-Armitage trend test.

Comparisons of allele frequency and genotype distribution of the rest SNPs are shown in Table S6.

$p$ values $<0.05$ are shown in bold.

$\mathrm{IFH}$, isolated fasting hyperglycemia; IPH, isolated postprandial hyperglycemia.

doi:10.1371/journal.pone.0071399.t004

(rs7756992 $p_{\text {trend }}=0.0122 ;$ rs8050136 $p_{\text {trend }}=0.0024 ;$ rs9939609 $p_{\text {trend }}=0.0049 ;$ rs780094 $\left.p_{\text {trend }}=0.0037\right)$. Allele frequencies or genotype distributions of $C D K N 2 B A S$, TP53INP1 and PRC1 were statistically comparable between IFH and IPH (Table S6).

\section{Discussion}

Current understanding on the genetic etiology of type 2 diabetes has been greatly improved over past decades. Considering the high heterogeneity of IFH and IPH, we proposed the hypothesis that genetic bases of IFH and IPH are different. Further, we speculated that genetic variants in loci modulating insulin sensitivity promoted IFH, while variants in loci affecting beta cell function promoted IPH.

The present study indicated that gene variants from FTO and $G C K R$ were specifically associated with increased risk of IFH, while gene variant from $C D K A L 1$ was associated with increased risk of IPH. It also showed that risk allele frequencies of FTO and $G C R K$ in IFH were higher than that in IPH. However, risk allele frequency of CDKAL1 in IFH was lower than that in IPH.

FTO was initially found to be associated with obesity in Caucasians [20]. In this study, we also confirmed the association of FTO with obesity (shown as WC and BMI) in newly diagnosed type 2 diabetes. It was reported that the fat mass and obesity associated protein, which is the genetic product of FTO, plays a role in the regulation of insulin sensitivity and insulin secretion from pancreatic beta cells [21]. Berulava et al. [22] found that SNP rs9939609 influenced the transcription of FTO. FTO SNPs rs8050136 and rs9939609, located in the same linkage disequilibrium block, were first found to be associated with type 2 diabetes in Caucasians [11,12]. According to Hapmap, risk allele (A) frequency of rs8050136 and rs9939609 are much higher in Caucasians (0.450), compared to East Asians (0.144). Studies in the Han Chinese population failed to establish consistency of association between FTO variants and type 2 diabetes [23-28]. Further, no association remained between FTO and type 2 diabetes after BMI adjustment in some studies, indicating that $F T O$ variants were associated with increased risk of type 2 diabetes through modulating the process of obesity development [25-27]. However, some reports do not support this viewpoint [23,24]. It had been well accepted that these contradictory findings result from the low MAF in Han Chinese, which results in insufficient statistical power $[23,26,28]$.
In the present study, we found that SNPs in FTO are associated with a $40 \%$ increased risk for IFH. Furthermore, we found OR decreased to $25-26 \%$ after adjustment for BMI $(p>0.05$, as the sample size of IFH was not sufficient large). On the other hand, SNPs in FTO were not associated with IPH (Table S5). We postulate that risk alleles of SNPs in FTO (rs8050136, rs9939609) or causal variants genetically linked with them may specifically promote risk of IFH in Han Chinese, which is partially independent of general obesity (evaluated by BMI).

Based on these observations, we speculate that previous findings of the association between FTO and type 2 diabetes may have resulted from the different proportions of IFH and IPH in those type 2 diabetes populations. According to DECODE and DECODA, the ratios of IFH/IPH were $613 / 473(40.4 \% /$ $28.4 \%)$ in Caucasians and 220/546 (18.1\%/44.9\%) in Asians $[3,29]$. In the CNDMDS performed in 2007-2008, the ratio of IFH/IPH was $17 \% / 47 \%$ (unpublished data) in Han Chinese. Previous studies have demonstrated that insulin resistance in Caucasians is much higher than Han Chinese, while Han Chinese typically have worse beta cell function than Caucasians [3,29]. Therefore, FTO is well established as a susceptibility gene of type 2 diabetes in Caucasians [11,12], but not in Chinese. No association between FTO and type 2 diabetes was observed in our population of newly diagnosed patients.

Glucokinase regulatory protein is the product of GCKR genetic encoding, whose overexpression could ameliorate insulin sensitivity and glucose tolerance in mice, while leading to higher serum TG [30]. GCKR gene polymorphisms were initially reported to be associated with TG levels [15]. In the present study, we confirm that the $\mathrm{G}$ allele of rs780094 in GCKR is associated with increased FPG and lower TG levels in newly diagnosed type 2 diabetes of Han ancestry (Table S3).

GCKR (rs780094) was first found to be associated with type 2 diabetes in Caucasians [15]. A nonsynonymous variant of GCKR (rs1260326, P446L) has strong linkage disequilibrium with rs780094 according to HapMap II data (CEU: $\mathrm{r}^{2}=0.93$; CHB/ JPT: $\left.r^{2}=0.83\right)$. This variant could regulate insulin secretion and blood TG levels through regulating the activity of glucokinase in the liver, while it was also correlated with type 2 diabetes [31]. In Han Chinese, the association of rs780094 with type 2 diabetes did not allow for consistent conclusions to be drawn [25,32-35]. Notably, we found that the $G$ allele was associated with increased 
risk of IFH, but decreased risk of IPH. Therefore, it's not surprising that we cannot confirm a correlation between rs780094 and newly diagnosed type 2 diabetes. We suppose that risk alleles of causal variants at the same linkage disequilibrium region with rs780094 specifically promote the progression of IFH. Therefore, the proportion of IFH and IPH adds to the debate over whether GCKR is a susceptibility gene for type 2 diabetes in Han Chinese.

Genetic product of the CDKAL1 gene belongs to the mammalian methylthyiotransferase family, and is involved in the process of synthesis and cleavage of proinsulin by ensuring the accurate translation of Lys codons [36]. It has been reported that the proinsulin/insulin conversion rate of CDKAL1 (rs7754840) risk allele carriers were lower [37]. Variations in CDKAL1 have been reported to be associated with impaired insulin secretion and the increased risk of type 2 diabetes. The association of CDKAL1 (rs7756992) with type 2 diabetes has been well established in Han Chinese [19,38,39]. In this study, we found that $G$ allele of rs7756992 was associated with increased risk of IPH, rather than IFH (Table S4). Though the sample size of IFH cases was smaller than that of IPH, allele frequencies and genotype distribution of rs7756992 were significantly different and the power to detect association of IFH and rs7756992 reached 79.49\% (Table S7). Moreover, it was reported that rs7756992, a common variant in CDKAL1 loci (MAF = 0.479), contributed to type 2 diabetes risk in Han Chinese in a study with a similar sample size [39]. Therefore, we speculate that the risk allele of rs7756992 was specifically associated with increased risk of IPH. Association of rs7756992 with newly diagnosed type 2 diabetes was also confirmed in present study, which likely resulted from the high proportion of IPH in the Han Chinese population.

We also observed that IFH and IPH share common susceptible SNPs including TCF7L2 and KCNQ1, both of which played approximately equal effect sizes in IFH and IPH subjects, respectively. TCF7L2 and $K C N Q 1$ were previously considered to be the strongest type 2 diabetes susceptible genes in Caucansians and East Asians [9,12-15,17-20,40-43], both of which were well replicated in Han Chinese [25,35,40,41,44,45]. Both TCF7L2 and $K C N Q 1$ have been shown to play essential roles in beta cell survival and function, and they have been suggested to be involved in the modulation of insulin sensitivity and obesity [42,43,46-49]. Based on our observations, causal variants in linkage disequilibrium with rs7903146 and rs2237895 were the common hereditary bases of IFH and IPH, resulting in their similar clinical features. In addition, $\mathrm{Yu}$, et al. demonstrated that the $\mathrm{C}$ allele of rs2237895 showed a greater effect on diabetes risk in participants with lower BMI, as well as a correlation between BMI and rs2237895 [50]. Our results, and other studies, support the idea that subjects carrying risk alleles at rs2237895 have lower BMI in newly diagnosed type 2 diabetes patients.

Though we confirm that $C D K N 2 B A S$ is associated with IFH, while PRC1 and TP53INP1 are associated with IPH, the allele frequencies of the three SNPs were not found to be different between IFH and IPH (Table S6). However, it should not be ignored that only $341 \mathrm{IFH}$ individuals were recruited, resulting in a lower or insufficient statistical power for IFH (Table S7). Further investigation of large samples at these loci is warranted to elucidate their association with IPH or IFH.

We observed that the five variants associated with IFH were additively associated with the increased risk of IFH. Moreover, six variants associated with IPH were additively associated with the increased risk of IPH. Despite of the common hereditary bases, different joint effects of SNPs on IFH and IPH groups also reflect hereditary differences.
The present study has some limitations to be addressed. First, the study could not achieve enough statistical power for several SNPs because of the limited sample size (in particular, the sample size of incident IFH was relatively small). Moreover, all SNPs involved in the study have been validated to be type 2 diabetes susceptible SNPs previously; thus novel loci could not be established by our work. However, this is the first study to investigate the susceptibility genes of IFH and IPH based on a general population sample which gave us samples of IFH and IPH. It also provides clues regarding targeted regions for future genetic research on IFH and IPH.

In conclusion, our study indicates that both distinct (FTO, GCKR, CDKAL1) and common genetic bases (TCF7L2, KCNQ1) exist for IPH and IFH in Han Chinese, suggesting specific underlying pathogenic mechanisms resulting in the heterogeneity of their clinic manifestation. Given the differences in risk allele frequencies, it will be valuable to further examine these genes thoroughly to search for the culprit disease loci of IPH and IFH. It also provides strong evidence for prediction, prevention, diagnosis, personalized medicine and drug development for Han Chinese with type 2 diabetes, among which most of the newly diagnosed type 2 diabetes patients have IPH.

\section{Supporting Information}

Table S1 Risk allele frequency, genotype distribution and Hardy-Weinberg equilibrium in study populations. ${ }^{\mathrm{a}}$ Risk alleles for type 2 diabetes in Caucasians are denoted in bold. Genotype distributions are shown as the counts of three genotypes $(\mathrm{BB}, \mathrm{Bb}$, bb). $\mathrm{B}$, risk allele; $\mathrm{b}$, non-risk allele. $p_{\mathrm{HWE}}<0.05$ is shown in bold, suggesting the SNP was not in Hardy-Weinberg equilibrium in the given population. IFH, isolated fasting hyperglycemia; IPH, isolated postprandial hyperglycemia.

(DOG)

Table S2 Association between SNPs and newly diagnosed type 2 diabetes in Han Chinese. ${ }^{a}$ Risk alleles for type 2 diabetes in Caucasians are denoted in bold. OR and 95\% CI are reported for the allele with higher type 2 diabetes risk as previously reported in Caucasians using $\chi^{2}$ or an additive model in logistic regression. ${ }^{b}$ Comparison of the allelic distribution between type 2 diabetes and controls. ${ }^{\mathrm{c}}$ Comparison of the genotype distribution between type 2 diabetes and controls after adjusting for region, age and gender. ${ }^{\mathrm{d}}$ Comparison of the genotype distribution between type 2 diabetes and controls after adjusting for region, age, gender and BMI. Empirical $p$ values were calculated through 1,000 permutations. $p$ values $<0.05$ are shown in bold.

(DOC)

Table S3 Association between SNPs and clinical features in control and newly diagnosed type 2 diabetes subjects. $\beta$ value is reported for the allele with higher type 2 diabetes risk as previously reported in Caucasians using an additive model in multi-variate linear regression adjusted for region, gender and age, with BMI when appropriate. Associations with $p$ value $<0.05$ are shown in the table. Empirical $p$ values were calculated through 1,000 permutations. Empirical $p$ values $<0.05$ are shown in bold.

\section{(DOC)}

Table S4 SNPs did not show significant association with isolated fasting hyperglycemia in Hans. ${ }^{\text {a }}$ Risk alleles for type 2 diabetes in the Caucasian descent population are denoted in bold. OR and 95\% CI are reported for the allele with higher type 2 diabetes risk previously reported for Caucasians using $\chi 2$ or an additive model in logistic regression. ${ }^{b}$ Comparison of the allelic distribution between isolated fasting hyperglycemia and controls. ${ }^{\mathrm{c}}$ Compar- 
ison of the genotype distribution between isolated fasting hyperglycemia and controls after adjusting for region, age and gender. $d$ Comparison of the genotype distribution between isolated fasting hyperglycemia and controls after adjusting for region, age, gender and $\mathrm{BMI}$. We failed to compare genotype distribution between isolated fasting hyperglycemia and controls at rs7957197 because its minor allele frequency of present samples was very low. Empirical $p$ values were calculated through 1,000 permutations. $p$ values $<0.05$ are shown in bold.

(DOC)

Table S5 SNPs did not show significant association with isolated postprandial hyperglycemia in Hans. ${ }^{a}$ Risk alleles for type 2 diabetes in the Caucasian descent population are denoted in bold. OR and 95\% CI are reported for the allele with higher type 2 diabetes risk previously reported for Caucasians using $\chi 2$ or an additive model in logistic regression. ${ }^{\mathrm{b}}$ Comparison of the allelic distribution between isolated postprandial hyperglycemia and controls. ${ }^{\mathrm{c}}$ Comparison of the genotype distribution between isolated postprandial hyperglycemia and controls after adjusting for region, age and gender. " Comparison of the genotype distribution between isolated postprandial hyperglycemia and controls after adjusting for region, age, gender and BMI. Empirical $p$ values were calculated through 1,000 permutations. $p$ values $<0.05$ are shown in bold.

(DOC)

Table S6 SNPs did not show significant differences in risk allele frequency or genotype distribution between isolated fasting hyperglycemia and isolated postprandial hyperglycemia. ${ }^{a}$ Risk alleles for type 2 diabetes in Caucasians are denoted in bold. Allelic frequencies between isolated fasting hyperglycemia and isolated postprandial hyperglycemia were compared using $\chi 2$ test. Genotype distributions are shown as the counts of three genotypes $(\mathrm{BB}, \mathrm{Bb}, \mathrm{bb}) . \mathrm{B}$, risk allele; b, non-risk allele. Genotype distributions between isolated fasting hyperglycemia and isolated postprandial hyperglycemia were compared using CochranArmitage trend test. $p$ values $<0.05$ are shown in bold. IFH,

\section{References}

1. Yang W, Lu J, Weng J, Jia W, Ji L, et al. (2010) Prevalence of diabetes among men and women in China. N Engl J Med 362: 1090-1101.

2. Adam JMF, Josten D (2008) Isolated post-challenge hyperglycemia: concept and clinical significance. Acta Med Indones 40: 171-175.

3. DECODE Study Group on behalf of the European Diabetes Epidemiology Study Group (1998) Will new diagnostic criteria for diabetes mellitus change phenotype of patients with diabetes? Reanalysis of European epidemiological data. BMJ 317: 371-375.

4. Meigs JB, Muller DC, Nathan DM, Blake DR, Andres R, et al. (2003) The natural history of progression from normal glucose tolerance to type 2 diabetes in the Baltimore longitudinal study of aging. Diabetes 52: 1475-1484.

5. Ceriello A (2003) The possible role of postprandial hyperglycaemia in the pathogenesis of diabetic complications. Diabetologia 46: M9-16.

6. Altshuler D, Hirschhorn JN, Klannemark M, Lindgren CM, Vohl MC, et al. (2000) The common PPARgamma Prol2Ala polymorphism is associated with decreased risk of type 2 diabetes. Nat Genet 26: 76-80.

7. Grant SF, Thorleifsson G, Reynisdottir I, Benediktsson R, Manolescu A, et al. (2006) Variant of transcription factor 7-like 2 (TCF7L2) gene confers risk of type 2 diabetes. Nat Genet 38: 320-323.

8. Sandhu MS, Weedon MN, Fawcett KA, Wasson J, Debenham SL, et al. (2007) Common variants in WFS1 confer risk of type 2 diabetes. Nat Genet 39: 951953.

9. Gudmundsson J, Sulem P, Steinthorsdottir V, Bergthorsson JT, Thorleifsson G, et al. (2007) Two variants on chromosome 17 confer prostate cancer risk, and the one in TCF2 protects against type 2 diabetes. Nat Genet 39: 977-983.

10. Sladek R, Rocheleau G, Rung J, Dina C, Shen L, et al. (2007) A genome-wide association study identifies novel risk loci for type 2 diabetes. Nature 445: 881885 .

11. Zeggini E, Weedon MN, Lindgren CM, Frayling TM, Elliott KS, et al. (2007) Replication of genome-wide association signals in UK samples reveals risk loci for type 2 diabetes. Science 316: 1336-1341. isolated fasting hyperglycemia; IPH, isolated postprandial hyperglycemia.

\section{(DOC)}

Table S7 Power of the association studies. ${ }^{\text {a }}$ Risk alleles for type 2 diabetes in Caucasians are denoted in bold. ${ }^{\mathrm{b}}$ ORs and 95\% CIs reported by reference studies (Ref.). ${ }^{\mathrm{c}}$ Power to detect the association between SNPs and newly diagnosed type 2 diabetes was estimated under the additive model and given the reported OR(95\%CI), sample size and $\alpha=0.05$ (two-sided). ${ }^{\mathrm{d}}$ Power to detect the association between SNPs and isolated fasting hyperglycaemia was estimated under the additive model and given the reported $\mathrm{OR}(95 \% \mathrm{CI})$, sample size and $\alpha=0.05$ (twosided). ${ }^{\mathrm{e}}$ Power to detect the association between SNPs and isolated postprandial hyperglycaemia was estimated under the additive model and given the reported $\mathrm{OR}(95 \% \mathrm{CI})$, sample size and $\alpha=0.05$ (two-sided). IFH, isolated fasting hyperglycemia; IPH, isolated postprandial hyperglycemia.

(DOC)

\section{Acknowledgments}

We thank all the patients for their cooperation in the study. We thank all the staff of CNDMDS (2007-2008) who contributed in recruiting participants and obtaining samples. We acknowledge the genotyping work of Beijing Genomics Institute, Shenzhen, China. We thank Prof. Cheng $\mathrm{Hu}$ and his group from Shanghai Diabetes Institute, Shanghai Key Laboratory of Diabetes Mellitus, Shanghai Jiaotong University Affiliated Sixth People's Hospital for their kind help in this study.

\section{Author Contributions}

Conceived and designed the experiments: WY JX XK. Performed the experiments: XK JH YC LC Z. Zhao QL JG GC XG J. Lu JW WJ LJJX ZS J. Liu HT QJ DZ Z. Zhou GS WY. Analyzed the data: XK JH. Contributed reagents/materials/analysis tools: WY LC Z. Zhao QL JG GC XG J. Lu JW WJ LJJX ZS J. Liu HT QJ DZ Z. Zhou GS. Wrote the paper: XK JH JX WY. Interpretation of data, critical review and approval of the final version of the manuscript to be published: XK JH YC LC ZZ QL JG GC XG JL JW WJ LJ JX ZS JL HT QJ DZ ZZ GS WY.

12. Scott LJ, Mohlke KL, Bonnycastle LL, Willer CJ, Li Y, et al. (2007) A genomewide association study of type 2 diabetes in Finns detects multiple susceptibility variants. Science 316: 1341-1345

13. Steinthorsdottir V, Thorleifsson G, Reynisdottir I, Benediktsson R, Jonsdottir T, et al. (2007) A variant in CDKAL1 influences insulin response and risk of type 2 diabetes. Nat Genet 39: 770-775.

14. Prokopenko I, Langenberg C, Florez J, Saxena R, Soranzo N, et al. (2009) Variants in the melatonin receptor 1B gene (MTNR1B) influence fasting glucose levels. Nat Genet 41: 77-81.

15. Diabetes Genetics Initiative of Broad Institute of Harvard and MIT (2007) Genome-wide association analysis identifies loci for type 2 diabetes and triglyceride levels. Science 316: 1331-1336.

16. Zeggini E, Scott LJ, Saxena R, Voight BF, Marchini JL, et al. (2008) Metaanalysis of genome-wide association data and large-scale replication identifies additional susceptibility loci for type 2 diabetes. Nat Genet 40: 638-645.

17. Unoki H, Takahashi A, Kawaguchi T, Hara K, Horikoshi M, et al. (2008) SNPs in KCNQ1 are associated with susceptibility to type 2 diabetes in East Asian and European populations. Nat Genet 40: 1098-1120.

18. Voight BF, Scott LJ, Steinthorsdottir V, Morris AP, Dina C, et al. (2010) Twelve type 2 diabetes susceptibility loci identified through large-scale association analysis. Nat Genet 42: 579-589.

19. Hu C, Zhang R, Wang C, Wang J, Ma X, et al. (2009) PPARG, KCNJ11, CDKAL1, GDKN2A-CDKN2B, IDE-KIF11-HHEX, IGF2BP2 and SLC30A8 are associated with type 2 diabetes in a Chinese population. PLoS ONE 4: e7643.

20. Frayling TM, Timpson NJ, Weedon MN, Zeggini E, Freathy RM, et al. (2007) A common variant in the FTO gene is associated with body mass index and predisposes to childhood and adult obesity. Science 316: 889-894.

21. Tung YC, Yeo GS (2011) From GWAS to biology: lessons from FTO. Ann N Y Acad Sci 1220: 162-171.

22. Berulava T, Horsthemke B (2010) The obesity-associated SNPs in intron 1 of the FTO gene affect primary transcript levels. Eur J Hum Genet 18: 1054-1056. 
23. Liu Y, Liu Z, Song Y, Zhou D, Zhang D, et al. (2010) Meta-analysis added power to identify variants in FTO associated with type 2 diabetes and obesity in the Asian population. Obesity 18: 1619-1624.

24. Han X, Luo Y, Ren Q Zhang X, Wang F, et al. (2010) Implication of genetic variants near SLC30A8, HHEX, CDKAL1, CDKN2A/B, IGF2BP2, FTO, TCF2, KCNQ1 and WFS1 in type 2 diabetes in a Chinese population. BMC Med Genet 11: 81.

25. Wen J, Rönn T, Olsson A, Yang Z, Lu B, et al. (2010) Investigation of type 2 diabetes risk alleles support CDKN2A/B, CDKAL1 and TCF7L2 as susceptibility genes in a Han Chinese cohort. PloS ONE 5: e9153.

26. Chang YC, Liu PH, Lee WJ, Chang TJ, Jiang YD, et al. (2008) Common variation in the fat mass and obesity-associated (FTO) gene confers risk of obesity and modulates BMI in the Chinese population. Diabetes 57: 2245-2252.

27. Ng MC, Tam CH, So WY, Ho JS, Chan AW, et al. (2010) Implication of genetic variants near NEGR1, SEC16B, TMEM18, ETV5/DGKG, GNPDA2, LIN7C/BDNF, MTCH2, BCDIN3D/FAIM2, SH2B1, FTO, MC4R, and KCTD 15 obesity and type 2 diabetes in 7705 Chinese. J Clin Endocrinol Metab 95: 2418-2425.

28. Li H, Wu Y, Loos RJ, Hu FB, Liu Y, et al. (2008) Variants in the fat mass- and obesity-associated (FTO) gene are not associated with obesity in a Chinese Han population. Diabetes 57: 264-268.

29. Qiao Q Nakagami T, Tuomilehto J, Borch-Johnsen K, Balkau B, et al. (2000) Comparison of the fasting and the 2-h glucose criteria for diabetes in different Asian cohorts. Diabetologia 43: 1470-1475.

30. Farrelly D, Brown KS, Tieman A, Ren J, Lira SA, et al. (1999) Mice mutant for glucokinase regulatory protein exhibit decreased liver glucokinase: a sequestration mechanism in metabolic regulation. Proc Natl Acad Sci USA 96: 1451114516.

31. Beer NL, Tribble ND, McCulloch LJ, Roos C, Johnson PR, et al. (2009) The $\mathrm{P} 446 \mathrm{~L}$ variant in the GCKR associated with fasting plasma glucose and triglyceride levels exerts its effect through increased glucokinase activity in liver. Hum Mol Genet 18: 4081-4088.

32. Qi Q, Wu Y, Li H, Loos RJ, Hu FB, et al. (2009) Association of GCKR rs780094, alone or in combination with GCK rs 1799884, with type 2 diabetes and related traits in a Han Chinese population. Diabetologia 52: 834-843.

33. Hu C, Zhang R, Wang C, Yu W, Lu J, et al. (2010) Effects of GCK, GCKR, G6PC2 and MTNR1B variants on glucose metabolism and insulin secretion. PLoS ONE 5: e11761.

34. Qi Q, Li H, Wu Y, Liu C, Wu H, et al. (2010) Combined effects of 17 common genetic variants on type 2 diabetes risk in a Han Chinese population. Diabetologia 53: 2163-2166.

35. Cui B, Zhu X, Xu M, Guo T, Zhu D, et al. (2011) A genome-wide association study confirms previously reported loci for type 2 diabetes in Han Chinese. PLoS ONE 6: e22353.

36. Wei FY, Suzuki T, Watanabe S, Kimura S, Kaitsuka T, et al. (2011) Deficit of tRNALys modification by Cdkall causes the development of type 2 diabetes in mice. J Clin Sci 121: 3598-3608.
37. Stančáková A, Kuulasmaa T, Paananen J, Jackson AU, Bonnycastle LL, et al. (2009) Association of 18 confirmed susceptibility loci for type 2 diabetes with indices of insulin release, proinsulin conversion, and insulin sensitivity in 5,327 nondiabetic Finnish men. Diabetes 58: 2129-2136.

38. Xu M, Bi Y, Xu Y, Yu B, Huang Y, et al. (2010) Combined effects of 19 common variations on type 2 diabetes in Chinese: results from two communitybased studies. PLoS ONE 5: e14022.

39. Wu Y, Li H, Loos RJ, Yu Z, Ye X, et al. (2008) Common variants in CDKAL1 CDKN2A/B IGF2BP2 SLC30A8 and HHEX/IDE genes are associated with type 2 diabetes and impaired fasting glucose in a Chinese Han population. Diabetes 57: 2834-2842.

40. Tsai FJ, Yang CF, Chen CG, Chuang LM, Lu CH, et al. (2010) A genome-wide association study identifies susceptibility variants for type 2 diabetes in Han Chinese. PLoS Genet 6: e1000847.

41. Hu C, Wang C, Zhang R, Ma X, Wang J, et al. (2009) Variations in KCNQ1 are associated with type 2 diabetes and beta cell function in a Chinese population. Diabetologia 52: 1322-1325.

42. Shu L, Sauter NS, Schulthess FT, Matveyenko AV, Oberholzer J, et al. (2008) Transcription factor 7-like 2 regulates beta-cell survival and function in human pancreatic islets. Diabetes 57: 645-653.

43. Rosengren AH, Braun M, Mahdi T, Andersson SA, Travers ME, et al. (2012) Reduced insulin exocytosis in human pancreatic beta cells with gene variants linked to type 2 diabetes. Diabetes 61: 1726-1733.

44. Liu Y, Zhou DZ, Zhang D, Chen Z, Zhao T, et al. (2009) Variants in KCNQ1 are associated with susceptibility to type 2 diabetes in the population of mainland China. Diabetologia 52: 1315-1321.

45. Lin Y, Li P, Cai L, Zhang B, Tang X, et al. (2010) Association study of genetic variants in eight genes loci with type 2 diabetes in a Han Chinese population. BMC Med Genet 11: 97

46. Le Bacquer O, Kerr-Conte J, Gargani S, Delalleau N, Huyvaert M, et al. (2012) TCF7L2 rs7903146 impairs islet function and morphology in non-diabetic individuals. Diabetologia 55: 2677-2681.

47. Chang YC, Chiu YF, Ho LL, Ting CT, Shih KC, et al. (2010) TCF7L2 genetic variants and progression to diabetes in the Chinese population: pleiotropic effects on insulin secretion and insulin resistance. J Mol Med 88: 183-192.

48. Yamagata K, Senokuchi T, Lu M, Takemoto M, Fazlul Karim M, et al. (2011) Voltage-gated $\mathrm{K}(+)$ channel KCNQ1 regulates insulin secretion in MIN6 $\beta$-cell line. Biochem Biophys Res Commun 407: 620-625.

49. Boini KM, Graf D, Hennige AM, Koka S, Kempe DS, et al. (2009) Enhanced insulin sensitivity of gene-targeted mice lacking functional KCNQ1. AmJ Physiol Regul Integr Comp Physiol 296: R1695-1701.

50. Yu W, Ma RC, Hu C, So WY, Zhang R, et al. (2012) Association between KCNQ1 genetic variants and obesity in Chinese patients with type 2 diabetes. Diabetologia 55: 2655-2659. 